In the second lecture, Altmann teaches us about quaternions (as well as, rather briskly, complex numbers) and how Hamilton failed to carry his celebrated vision on Brougham Bridge through to its proper conclusion. If $\varnothing$ rsted confused an axial with a polar vector, Hamilton's mistake was to confuse it with a 180 degree rotation about that axis. As a result it was left to Rodriguez to derive with complicated spherical geometry what Hamilton ought to have found trivially with his quaternions: the relation between the product of two rotations and their half angles. If Ørsted was deluded by taking his arrow too literally, Hamilton fell under the spell of his quaternion units $i, j$ and $k$, whose squares were -1 , like the square of the complex unit $i$. He thus could not resist associating them with 90 degree rotations, thereby missing their true character as rotations through 180 degrees.

In the final and most ambitious lecture, Altmann discusses the instability of a one-dimensional conducting crystal against spontaneously deforming into an insulating crystal with a larger unit cell. The prediction of this now celebrated effect existed for many years only as a parenthetical aside in Rudolf Peierls's wonderful textbook on solid-state physics. To describe the Peierls transition to his general readers, Altmann develops in a few pages the concepts of energy levels, degeneracies, orbitals, Brillouin zones and much of the other apparatus of the independent electron theory of metals, even proving that a glide plane implies a twofold degeneracy at the zone boundary. It is a heroic and highly original effort, which I shall certainly return to for inspiration should I ever feel courageous enough to make such an attempt myself. But undergraduates in the United States, sixth-formers and even mature scientists who have not survived a course in quantum mechanics may well find it puzzling.

In launching this pedagogical tour de force, Altmann remarks that "it is very easy to talk rubbish when discusssing degeneracy and, even if one is not aiming at much rigour, a minimum of decency in the treatment is absolutely essential if one is not to fall into nonsense." Anyone presenting serious science to the nonspecialist would be well advised to take to heart the general principle enunciated here. Altmann has made a first-class effort in these essays, rarely sacrificing accuracy for the illusion of intelligibility, always writing with good humour and grace, and maintaining considerable decency in the treatment.

N. David Mermin is in the Laboratory of Atomic and Solid State Physics, Cornell University, Ithaca, New York 14853-2501, USA.

\section{Source book}

\section{Andrew Miller}

Macromolecular Crystallography with Synchrotron Radiation. By John R. Helliwell. Cambridge University Press: 1992. Pp. 595. £95, $\$ 165$.

'HAve crystals, will travel.' This is a handbook for gipsy crystallographers seeking the brightest X-ray beams. Most of what we know about the molecular machinery of life comes from X-ray crystallography. When a biological macromolecule (enzyme, antibody, transfer RNA) or a macromolecular complex (virus, ribosome) can be crystallized, there is the potential, by X-ray crystallography, to determine the positions of all its atoms and hence to understand how it functions. The scattering power of these crystals is low and the crystals themselves are often small, so the brightest X-ray beams are required. The European Synchrotron Radiation Facility, which begins operating in 1993 and becomes available to users in 1994, will provide the brightest continuous X-ray beam in the world. John Helliwell has now written a valuable comprehensive survey of this research field.

Helliwell has adopted a catch-all approach to his wide-ranging subject. There are sections on methods of crystallization, elementary principles of macromolecular structure, basic crystal symmetry, the elements of X-ray diffraction, details of specific methods such as the Laue technique and multiple wavelength anomalous diffraction, time-resolution and molecular dynamics. There are chapters on the nature of synchrotron radiation, on storage rings, and on the range of sources that can now be designed, such as insertion devices, wigglers, wavelength shifters and tapered, tunable undulators. Beamlines receive $\mathrm{X}$ rays from a specific source and then condition the beam for use, and the beamline components, such as mirrors, monochromators and detectors, are also described. The subjects of many of these chapters could, and often do, have books to themselves. Helliwell has provided a valuable service by gathering such a diversity of material in one volume. The book will be a boon to final-year research students completing their theses (although few will be able to afford it).

Adequate theoretical foundations are provided throughout, but this is really an experimentalist's book, with emphasis on the practical details of working at synchrotron radiation sources. Part of the book is a sort of consumer's guide to synchrotron sources of the world, with lists of the beamlines available and their physical parameters. The practical details, which all too frequently are impossible to find, are particularly helpful to a newcomer to synchrotron radiation, and the assured tone of these sections is due to the fact that Helliwell has been involved in many experiments, justifying what might otherwise seem to be a surfeit of self-references. There are excerpts from synchrotron radiation laboratory manuals and helpful tables.

The book is written in a clear and simple, if somewhat breathless, style, with jargon defined and explained. It does not have the intellectual coherence of works by A. Guinier (X-ray Diffraction, Freeman) or W. Cochran and $\mathrm{H}$. Lipson (The Crystalline State, Vol. 3, Bell) - the field is too new for that. And advances in synchrotron radiation research are occurring so rapidly that even this book, which has references to 1991 publications, is already out of date, but not damagingly so and certainly not through any fault of the author. Helliwell occasionally overreaches himself in his effort to be fully comprehensive; for example, there is only about half a page on the complex subject of inelastic neutron scattering in the investigation of molecular dynamics.

The unsung heroes of many of the spectacular results coming from synchrotron radiation are the scientific staff at the facilities who often show imagination and originality in building instruments that cope with extremes of radiation and that still perform superbly. Protein crystallographers rely on these innovators as much as on the biochemists who provide the crystals, and Helliwell's book gives them the full recognition they deserve.

Andrew Miller is in the Department of Biochemistry, University of Edinburgh, Edinburgh EH8 9XD, UK.

\section{New In paperback}

- Primate Paradigms: Sex Roles and Social Bonds by Linda Marie Fedigan, now with a new introduction. "Courageous and hard-hitting" was how John $\mathrm{H}$. Cook described this work in his review in Nature 302, 359 (1983). University of Chicago Press, $\$ 21.75, £ 15.25$.

- Changing Order: Replication and Induction in Scientific Practice by $\mathrm{H}$. M. Collins, now with a new afterword. Reviewed in Nature 319, 274 (1986). - The Holy Land: An Archaeological Guide from Earliest Times to $1700,3 r d$ Edn by Jerome Murphy-O'Conner. Reviewed in Nature 288, 503 (1980). Oxford University Press, $£ 8.99$.

- The Dark Romance of Dian Fossey by Harold Hayes, a compelling biography of the woman who abandoned her career as an occupational therapist to live in Africa among the last remaining mountain gorillas. Corgi Books, £6.99. 